Check for updates

Cite this: RSC Adv., 2019, 9, 6356

\title{
Highly crystalline and uniform conjugated polymer thin films by a water-based biphasic dip-coating technique minimizing the use of halogenated solvents for transistor applications $\uparrow$
}

\author{
Eun Hye Kwon, ${ }^{a}$ Young Jin Jang, ${ }^{a}$ Gun Woo Kim, ${ }^{\text {b }}$ Min Kim ${ }^{* c}$ \\ and Yeong Don Park (iD *ab
}

\begin{abstract}
The commercialization of organic electronics will require minimizing the use of halogenated solvents used to solution-process organic semiconductors, which is a crucial step for large-area coating methods, such as the dip-coating method. Here, we report a novel biphasic dip-coating method which uses a water-based biphasic solution and produces a uniform, smooth, and crystalline conjugated polymer thin film in the presence of a solvent additive. We demonstrated that a solvent additive with a high boiling point and solubility parameter similar to that of the solution affected the solvent evaporation rate and improved the crystallinity of the dip-coated polymer thin film. The method used to add the solvent strongly influenced how the solvent additive diffused into the polymer solution, which affected the resulting film morphology. The crystallinity and morphology of the polymer films were correlated with the electrical characteristics, and the most crystalline film displayed a high hole field effect mobility of $0.0391 \mathrm{~cm}^{2} \mathrm{~V}^{-1} \mathrm{~s}^{-1}$ when processed from the solvent mixture without post-treatment. Our findings provide a direction for the development of reliable and promising organic thin film transistor technologies.
\end{abstract}

Received 8th November 2018 Accepted 5th February 2019

DOI: $10.1039 / c 8 r a 09231 a$

rsc.li/rsc-advances conjugated semiconductor structures. ${ }^{12}$ The environmental impact of halogenated solvents becomes a major issue when fabricating large-area organic semiconductor coatings. ${ }^{13}$ The fabrication of high-mobility TFT devices using environmentally benign non-halogenated solvents remains a challenging and urgent task in this field.

Various solution processing methods have been developed for the fabrication of large-area coatings. ${ }^{7}$ Among these, dipcoating is widely employed in both academic research and industrial production. ${ }^{14,15}$ This method is advantageous for the preparation of uniform and smooth thin films on various curved flexible substrates as well as for providing fine control over conjugated polymer crystallization processes that empower efficient charge carrier transport. ${ }^{16-20}$ The dip-coating process is not readily scaled-up, however, because a large volume of the polymer solution is required to fill the dip-coating reservoir. To overcome this problem, a novel biphasic dip-coating method was developed using a phase-separated solvent system composed of a reusable inert liquid support phase (the majority of the system) and the solution to be deposited, floating on top of the support phase. ${ }^{21-23}$ In this configuration, the volume of the polymer solution can be greatly minimized.

Biphasic dip-coating processing requires the selection of a pair of solvents that can form a stabilized biphasic state. Typically, biphasic systems can be produced using a floating solvent with a low density and an underlying support solvent
Incheon 22012, Republic of Korea. E-mail: ydpark@inu.ac.kr

${ }^{b}$ Innovation Center for Engineering, Incheon National University, Incheon 22012, Republic of Korea

${ }^{c}$ Center for Nano Science and Technology@Polimi, Istituto Italiano di Tecnologia, Via Giovanni Pascoli 70/3, 20133 Milan, Italy.E-mail: min.kim@iit.it

$\dagger$ Electronic supplementary information (ESI) available. See DOI: 10.1039/c8ra09231a 
with a high density; ${ }^{22}$ however, most solvents that can dissolve $\pi$-conjugated polymers have a high density exceeding $1.1 \mathrm{~g} \mathrm{~cm}^{-3}$, making it difficult to find suitable underlying solvent phases. ${ }^{24}$ To reduce the environmental impact of dipcoating processes, it is important to find green solvents that can form stable biphasic states with organic semiconductor solutions. ${ }^{23}$ Here, we report a novel biphasic dip-coating method that uses water as a reusable bottom-phase solvent. We systematically studied the formation of the biphasic solution system, varying the solvent properties, including the solubility parameter and surface tension. To achieve a highly crystalline polymer film, we controlled the solvent evaporation rate by adding a solvent additive with a high boiling point, which strongly affected the polymer crystallization process. The solvent mixture biphasic system produced uniformly and smoothly dip-coated films containing a highly crystalline conjugated polymer phase for use in thin film transistor devices.

\section{Experimental section}

\subsection{Preparation of thin films and OTFT devices}

Poly(3-hexylthiophene) (P3HT, MW $=69 \mathrm{kDa}$, regioregularity $=96 \%$, PDI $=2.0-2.3)$ was purchased from Rieke Metals, Inc. P3HT was dissolved in chloroform (CF), chlorobenzene (CB), and dichlorobenzene (DCB) to a concentration of $7 \mathrm{mg} \mathrm{mL}^{-1}$ and was stirred at $50{ }^{\circ} \mathrm{C}$ for $3 \mathrm{~h}$. A highly doped n-type silicon wafer covered with a $300 \mathrm{~nm}$ thick thermally grown silicon dioxide $\left(\mathrm{SiO}_{2}\right)$ layer was submerged into a hot piranha solution (3: 2 volume mixture of $98 \%$ sulfuric acid and $35 \%$ hydrogen peroxide) for $30 \mathrm{~min}$ at $70{ }^{\circ} \mathrm{C}$ to remove organic contaminants. Octyltrichlorosilane (OTS) was coated by dipping the $\mathrm{Si} / \mathrm{SiO}_{2}$ substrates into toluene containing the OTS reagents for $30 \mathrm{~min}$ at room temperature. The OTS-treated substrate was ultrasonicated in toluene and ethanol for $3 \mathrm{~min}$ each and dried under vacuum prior to use. A $3 \mathrm{~mL}$ glass vial filled with $2.5 \mathrm{~mL}$ deionized (DI) water was used to form the support solvent, and $0.07 \mathrm{~mL}$ of the P3HT solution was carefully dropped onto the DI water surface using a syringe. The OTS-treated $\mathrm{Si} / \mathrm{SiO}_{2}$ substrate was dip-coated at a dip and withdrawal speed of $5 \mathrm{~mm} \mathrm{~s}^{-1}$ (EF-4100, E-flex). P3HT films were thermally treated at $80^{\circ} \mathrm{C}$ for $30 \mathrm{~min}$ and were kept under vacuum for 2 hours to evaporate the DI water and organic solvents prior to electrical characterization. Bottom-gate, top-contact organic TFT devices were fabricated by evaporating gold electrodes. A shadow mask with $1000 \mu \mathrm{m}$ channel widths and $100 \mu \mathrm{m}$ channel lengths was used to pattern the gold source and drain electrodes. The UV-vis absorption measurements were conducted using a P3HT film fabricated on OTS-treated glass substrate.

\subsection{Characterization}

UV-vis absorption spectra were measured using a UV-vis spectrophotometer (Thermo Scientific, Genesys 10S). The film morphologies were characterized by optical microscopy (OM, Olympus BX51) and atomic force microscopy (AFM, Multimode
8 , Bruker). The electrical properties of the P3HT film were obtained using a semiconductor analyzer (Keithley 4200-SCS) under vacuum at room temperature to prevent other effects. ${ }^{24}$ The field effect mobility was calculated in the saturation regime.

\section{Results and discussion}

\subsection{Formation of a biphasic system}

We chose poly(3-hexylthiophene) (P3HT) as a conjugated polymer, one of the most well-studied model systems for film formation and crystallization behavior. A biphasic dip-coating method was used comprising a top phase polymer solution that dissolved the organic semiconductor for deposition and a bottom phase solvent that filled most of the volume of the dipcoating container. The solvent properties of the top and bottom phase were varied to understand which properties contributed to the formation of a stable biphasic state. The majority of solvents that dissolved the conjugated polymers used in organic electronics are chlorinated solvents with high densities exceeding $1.1 \mathrm{~g} \mathrm{~cm}^{-3}\left(\mathrm{CF}=1.48 \mathrm{~g} \mathrm{~cm}^{-3}, \mathrm{CB}=1.11 \mathrm{~g} \mathrm{~cm}^{-3}, 1.2-\right.$ $\left.\mathrm{DCB}=1.30 \mathrm{~g} \mathrm{~cm}^{-3}\right) \cdot{ }^{25}$ Therefore, the candidates for the bottom phase solvent were significantly limited to solvents having a density higher than that of the top phase solution. In this study, we investigated other solvent properties, including the surface tension, $\gamma$, in an attempt to identify a suitable bottomphase green solvent for use in the stable biphasic system. Surface tension can be defined as the property of the surface of a liquid that allows it to resist an external force. ${ }^{26}$ The surface tension depends on the cohesive nature of the solvent molecules. A sufficiently high surface tension in the bottom phase solvent can assist biphasic solution formation, even if the solvent density of the top phase solution is higher than that of the bottom phase. We compared water with other two organic solvents with a surface tension lower than that of water to observe whether any of these solvents could form a biphasic solution state. Fig. 1 shows that only DI water formed a biphasic solution configuration with $\mathrm{P} 3 \mathrm{HT}$ in CF solution as the top phase, despite the fact that the density of water, $1 \mathrm{~g} \mathrm{~cm}^{-3}$, is lower than that of the polymer-dissolving solvent, $1.48 \mathrm{~g} \mathrm{~cm}^{-3} \cdot{ }^{25}$ Tetrahydrofuran (THF) and dimethylformamide (DMF), which have densities similar to that of DI water, displayed collapsed biphasic states because their surface tensions were insufficiently high.

\subsection{The secondary solvent addition process}

During the dip-coating process, the solvent properties of the top phase solution strongly affected the resulting film formation as well as the crystallization behavior of the conjugated polymer. ${ }^{27,28}$ The solvent evaporation rate is one of the most critical factors controlling the crystallinity of a polymer film prepared by dip coating. A high boiling point solvent can facilitate crystallization among polymer chains by prolonging the solvent evaporation time..$^{29,30}$ In this study, we tested three different solvents, CF, CB, and DCB, as top phase solvents with different boiling points, from 61 to $180^{\circ} \mathrm{C}$. The UV-vis spectra of the dipcoated films formed from the P3HT-water biphasic system were 
(a)
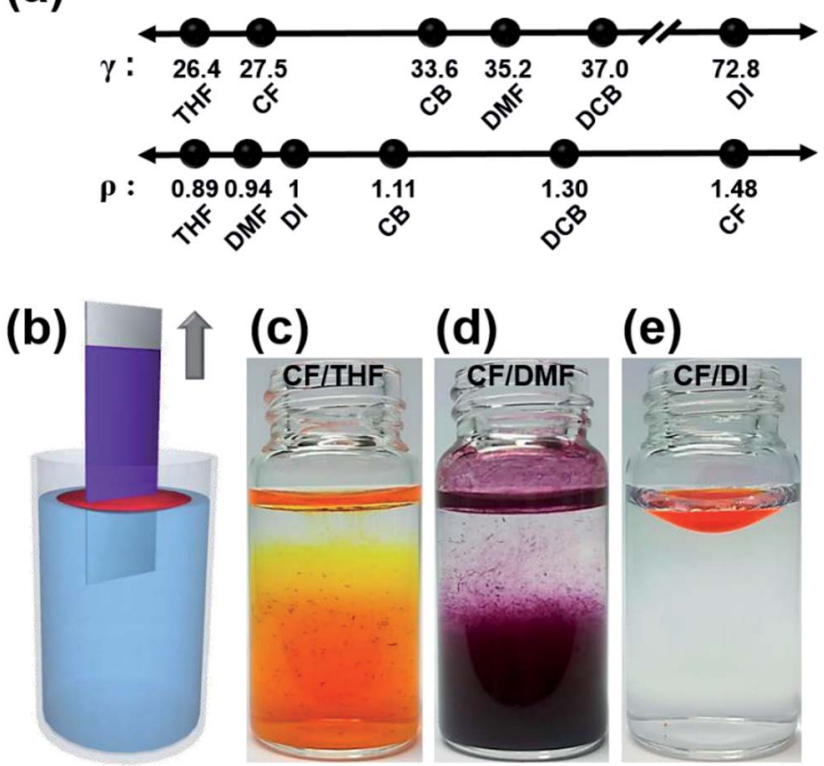

(c)

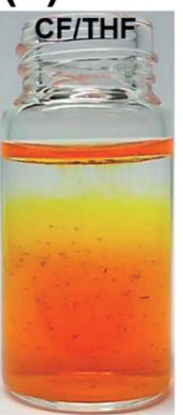

(d)

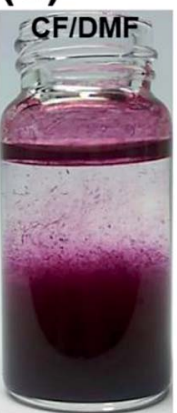

(e)

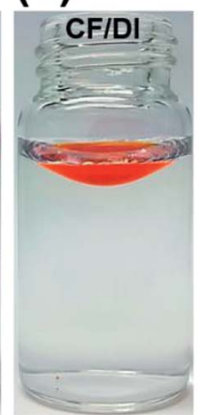

Fig. 1 (a) Surface tensions and densities of various solvents. (b) Schematic diagram illustrating the biphasic dip-coating process. Photographs of biphasic systems comprising (c) CF (top)/THF (bottom), (d) CF (top)/DMF (bottom), and (e) CF (top)/DI water (bottom).

compared (Fig. 2). The absorption spectra of the P3HT thin films prepared in $\mathrm{CF}, \mathrm{CB}$, and DCB showed absorption peaks characteristic of the P3HT thin films at 560 and $608 \mathrm{~nm}$ due to intermolecular interactions (Fig. 2a). The ratio of the intensity of the (0-0) peak at $608 \mathrm{~nm}$ to that of the $(0-1)$ peak at $560 \mathrm{~nm}$, $A_{0-0} / A_{0-1}$, was correlated with the degree of crystallinity in the P3HT film. ${ }^{31,32}$ Fig. 2e reveals that $A_{0-0} / A_{0-1}$ increased from 0.65 to 0.82 as the solvent boiling point increased. Although the DCB-processed P3HT film provided the highest degree of crystallinity, an excessively low solvent evaporation rate could decrease the film thickness and dewetting behavior due to solution agglomeration, discussed in greater detail in Section 3.3. ${ }^{33}$ We incorporated 1,2,4-trichlorobenzene, which has a high boiling point, $214{ }^{\circ} \mathrm{C}$, into our biphasic dip-coating system, and confirmed that an excessively high boiling point induced strong dewetting problems and resulted in poor film formation.

To reliably fabricate the $\mathrm{P} 3 \mathrm{HT}$ films, we incorporated a solvent additive into the polymer solution to control the solvent evaporation rate while preserving the uniform and pinhole-free film coverage. Numerous studies have reported that solvent additive-assisted solution processes can perform better than systems prepared without a solvent additive. ${ }^{27,34,35}$ We tested solvent additives in the biphasic dip-coating method in two different ways (Fig. 2b): (1) by floating the solvent additive on a polymer-dissolving top-phase solution (floating method); and (2) by mixing the solvent additive with the polymerdissolving solution before forming a biphasic system (mixing method). We used CB and DCB as solvent additives, and CF as the polymer-dissolving host solvent. First, we applied $\mathrm{CB}$ as a solvent additive to the P3HT-CF solution using the floating method. The absorption spectra of the P3HT films dip-coated from CF:CB exhibited strongly enhanced intermolecular peaks (a)

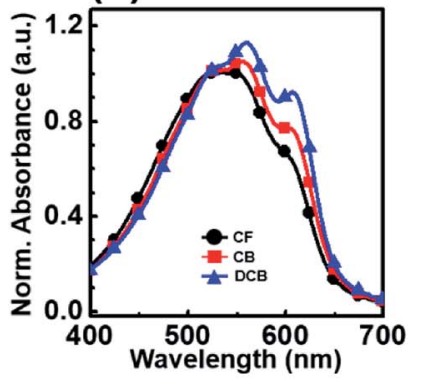

(c)

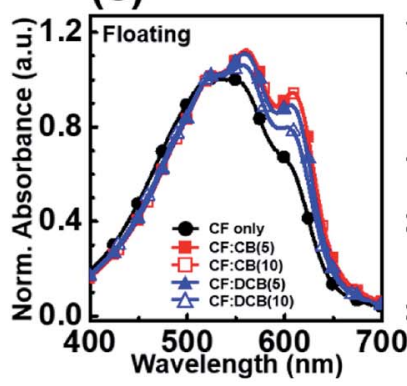

(b)

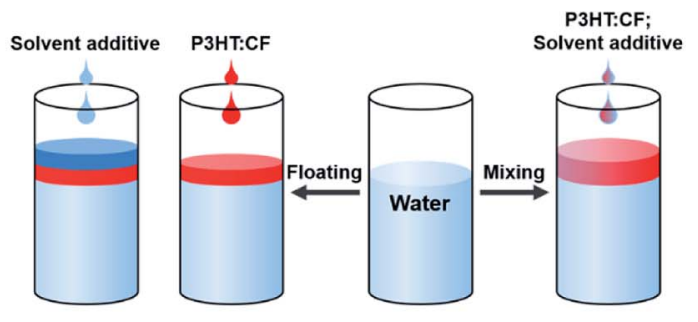

(d)

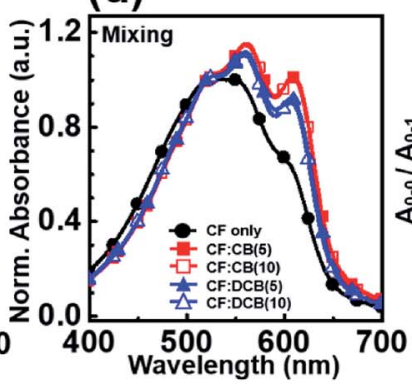

(e)

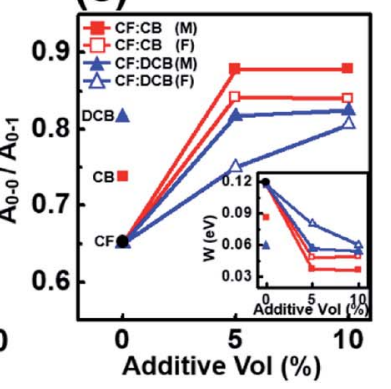

Fig. 2 (a) Normalized UV-vis absorption spectra of the P3HT thin films prepared from CF, CB, and DCB. (b) Schematic illustration of the solvent adding processes: the floating method and the mixing method. Normalized UV-vis absorption spectra of the P3HT thin films prepared from different solvent adding processes: (c) floating and (d) mixing method at a certain amount of additive solvent, 5 and 10 vol\%. (e) The ratios of the intensities of the $(0-0)$ to $(0-1)$ peaks. The inset shows variations in the free exciton bandwidth $W$, calculated from the UV-vis absorption spectra of the dip-coated P3HT thin films. 
at 560 and $608 \mathrm{~nm}$ compared to the films dip-coated from the homogenous solvent, CF (Fig. 2c). Even with the addition of small amount (5 vol\%) of $\mathrm{CB}$, the absorbance spectra show a remarkable increase in $A_{0-0} / A_{0-1}$ and reached saturation. Beyond the saturation point, the CF:CB (CB 10 vol\%) system provided a similar spectrum with pronounced (0-0) and (0-1) peak absorptions compared to those obtained from CF:CB (CB 5 vol\%); however, the absorption spectrum obtained from the CF:DCB solvent mixture showed a lower intensity at the (0-0) absorption peaks than was obtained from CF:CB, indicating that the CF:DCB films had a lower crystallinity than the CF:CB films. The absorption spectra of the CF:DCB films exhibited a strong dependency of the $A_{0-0} / A_{0-1}$ peak ratio on the volume ratio of DCB to $\mathrm{CB}$ (Fig. 2e). We hypothesized that the difference between the CF:CB and CF:DCB systems originated from the miscibility of the main and additive solvents, which affected how the solvent additive diffused into the host solution and solute, facilitating solute crystallization in the solution state. We confirmed that the Hansen solubility parameters of CB and CF were similar, which enabled the floating solvent additive CB to mix with the polymer-dissolving solution $\mathrm{CF}$, lowering the evaporation rate during film formation and enhancing the crystallinity of the resulting $\mathrm{P} 3 \mathrm{HT}$ film. ${ }^{36}$ The DCB solvent additive, which has a Hansen solubility parameter that is less similar to that of $\mathrm{CF}$, appeared to ineffectively diffuse into the P3HT solution and negligibly assisted P3HT crystallization compared to the CF:CB system. The differences between the CB and DCB additive properties revealed that good miscibility between the solvent and additive is critical for inducing P3HT crystallization using the solvent additive floating method.

The effects of the solvent additive mixing method on P3HT crystallization were further studied by adding the solvent additive, CB or DCB, to the polymer solution, P3HT/CF, before forming the biphasic solution. The $\mathrm{P} 3 \mathrm{HT}$ film processed from the solvent additive mixed solution showed more pronounced intermolecular (0-0) peaks compared to the P3HT film processed using the solvent additive floating solution. For both the CF:CB and CF:DCB systems, mixing the solvent additive with the P3HT solution provided a higher $A_{0-0} / A_{0-1}$ and a saturation trend using $5 \mathrm{vol} \%$ solvent additive. The $A_{0-0} / A_{0-1}$ peak ratio in the absorption spectra obtained from CF:DCB showed a much weaker dependence on the solvent additive volume ratio than did the ratio obtained from the CF:DCB floated solution. Pre-mixing the DCB solvent additive appeared to form a better mixed phase than the solvent additive floating method (Fig. 2e). These results revealed that the method used to incorporate a solvent additive into a P3HT solution strongly affected the P3HT crystallization behavior and the resulting film morphology.

\subsection{Film morphology}

The film coverage of the substrates was examined by acquiring photographs of the P3HT films dip-coated from biphasic solutions onto OTS-treated silicon substrates (Fig. 3). The solvent evaporation rate of the polymer-dissolving top-phase solution strongly affected the resulting film coverage. Lower solvent evaporation rates produced lower film coverage. We found that

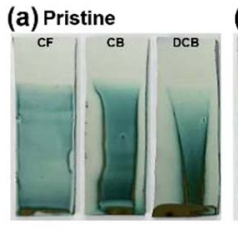

(d) Floating
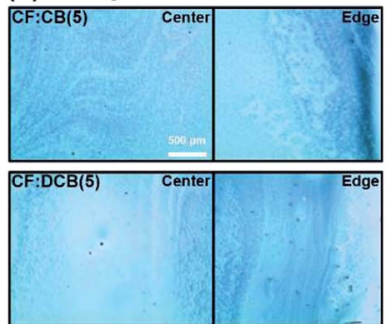

(b) Floating

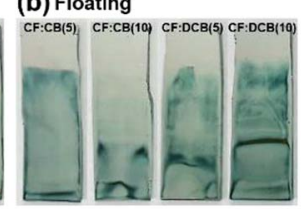

(c) Mixing

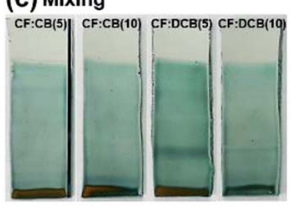

(e) Mixing
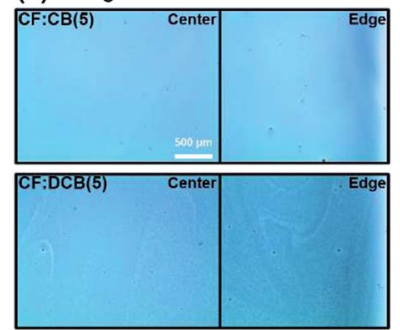

Fig. 3 Photographs of P3HT films dip-coated from the homogeneous solvent, (a) CF, CB, and DCB, and from the mixed solvents prepared using different solvent addition processes: (b) floating and (c) mixing method, onto an OTS-treated silicon substrate. Optical microscopy images of the P3HT films dip-coated from the biphasic solvents with solvent additives $C F: C B$ and $C F: D C B$ for the two different solvent addition methods: (d) the floating method and (e) the mixing method.

the solvent with a higher boiling point (CB or DCB) produced inhomogeneous film coverage with dewetted regions on the dipcoated P3HT film (Fig. 3a). This behavior arose from the dipcoating process, in which the solvent evaporation equilibrium and the drawing speed determined the film thickness and coverage (Fig. S1 $\dagger$ ). ${ }^{37}$ The high boiling point of DCB, $180{ }^{\circ} \mathrm{C}$, prolonged solvent evaporation and P3HT solidification, affording the solutes a longer time to reach thermodynamically stable states. This mobility induced film dewetting of the polymer semiconductor materials. ${ }^{29}$

To overcome the dewetting issue, we used solvent additives, CB or DCB, in the P3HT-CF solution. We obtained various film morphologies, depending on the solvent addition method. The
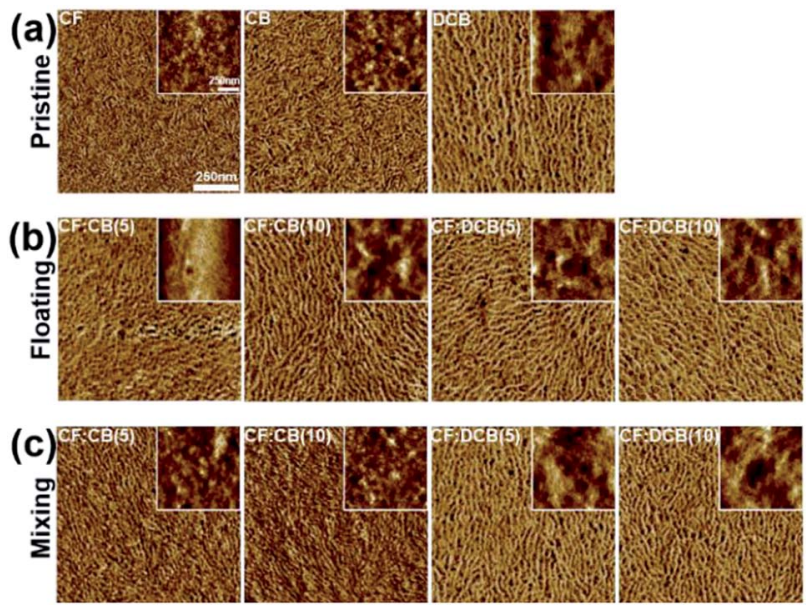

Fig. 4 Tapping-mode AFM phase images of the P3HT films obtained from the biphasic P3HT solutions. AFM phase images of the films processed from the solvents (a) CF, CB, and DCB. AFM phase images of the films processed from the solvent mixtures $C F: C B$ and $C F: D C B$ prepared using different solvent addition methods: (b) floating method and (c) mixing method. 
(a)

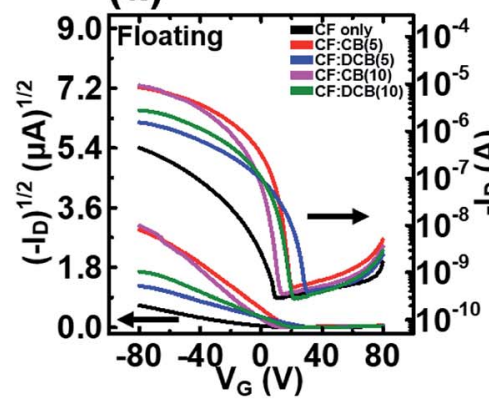

(b)

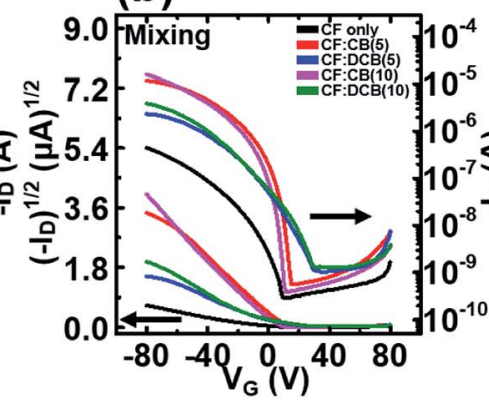

(c)

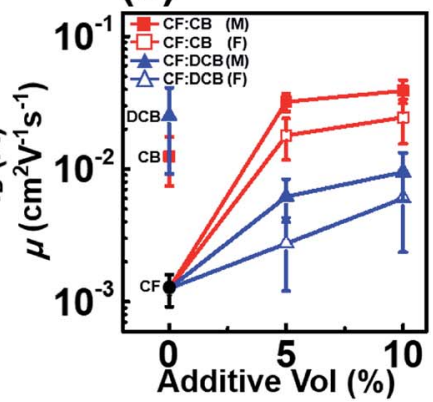

Fig. 5 Plots of the drain current versus the gate voltage at a fixed drain voltage of $-80 \mathrm{~V}$ on both the linear (left axis) and logarithmic (right axis) scales for P3HT films dip-coated from the solvent mixtures CF:CB and CF:DCB, prepared by (a) the floating method and (b) the mixing method. (c) Average field effect mobilities of the P3HT films dip-coated from the various solvent mixtures using various solvent addition methods.

solvent additive floating method prevented dewetting but rendered uneven films, regardless of the solvent additive (CB or DCB). The wave patterns in the P3HT film were generated by unstable solvent evaporation during the dip-coating process (Fig. 3b), possibly due to the inhomogeneous mixing and distribution of the solvent additive in the polymer solution. By contrast, the solvent additive mixing method introduced an additive to the polymer solution prior to floating and successfully rendered uniform and homogeneous films on the OTStreated silicon substrates (Fig. 3c). The CB and DCB additives, at volume ratios in the range of $5-10 \%$, produced full-coverage uniform P3HT films without defective wavy patterns.

The dip-coated films prepared from biphasic solutions with solvent additives were examined using optical microscopy (OM). The CF:CB and CF:DCB films processed using the solvent additive floating method contained P3HT aggregates and hardly achieved a uniform film morphology (Fig. 3d). These aggregates were abundant near the edge of the film, rendering the film non-uniform and unfavorable for TFT device applications. The film dip-coated from the solvent additive mixing method using $\mathrm{CF}: \mathrm{CB}$ as the additive solvent showed a clean homogeneous film with a smooth surface topology that was desirable for large-area device applications (Fig. 3e). On the other hand, the film processed from CF:DCB contained a wavy pattern that was not discernable in the photographs but was observable in the magnified OM images (Fig. 3c).

Atomic force microscopy (AFM) was used to determine the nanoscale morphologies of the dip-coated P3HT thin films (Fig. 4). The AFM images clearly revealed that the dip-coated P3HT film prepared from the high boiling point solvents contained well-ordered thick nanowires, whereas the film processed from the low boiling point solvent (CF) contained smaller crystalline phases. The larger nanowire size obtained from the neat CB- or the neat DCB-processed film indicated a highly crystallized film resulting from the low solvent evaporation rate. These results agreed well with the UV-vis results, which showed that P3HT crystallization during the biphasic dip-coating process was strongly affected by the solvent boiling point.

Addition of a solvent additive with a boiling point higher than that of the main solvent changed the surface morphology of the as-cast P3HT film. As the high boiling point solvent additive, $\mathrm{CB}$ or DCB, was added, overall the P3HT nanowires became thicker compared to the films processed from the neat CF solvent. Regardless of the volume ratio of the high boiling point solvent additive, adding even a small amount of solvent additive resulted in strong P3HT crystallization and was more effective than using the high boiling point homogeneous solvent. We hypothesized that the main solvent, CF, evaporated first to uniformly wet the substrates, and the remaining $\mathrm{CB}$ or DCB enabled the P3HT solution cast onto the substrate to fully self-assemble during solidification. These results indicated that adding a high boiling point solvent to the P3HT solution effectively assisted the formation of uniform films with a highly crystalline structure due to the low evaporation rate of the high boiling point solvent.

\subsection{Device characteristics}

The electrical characteristics of the P3HT films dip-coated from the homogeneous or mixed solvents were investigated by measuring the field effect mobilities of the P3HT films in TFTs with a top-contact transistor geometry. The typical drain current $\left(I_{\mathrm{D}}\right)$ vs. gate voltage $\left(V_{\mathrm{G}}\right)$ plots collected in the accumulation mode are shown in Fig. 5 . The addition of a high boiling point solvent to the polymer solution gradually increased the on-

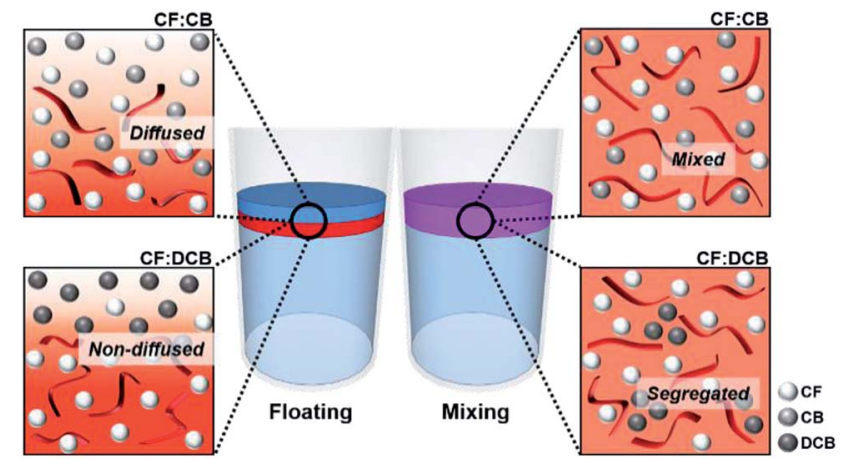

Fig. 6 Schematic drawing illustrating how the solvent addition method (floating and mixing) induced solvent intermixing and agglomeration in the solution state. 
Table 1 Summary of the various solvents and P3HT properties

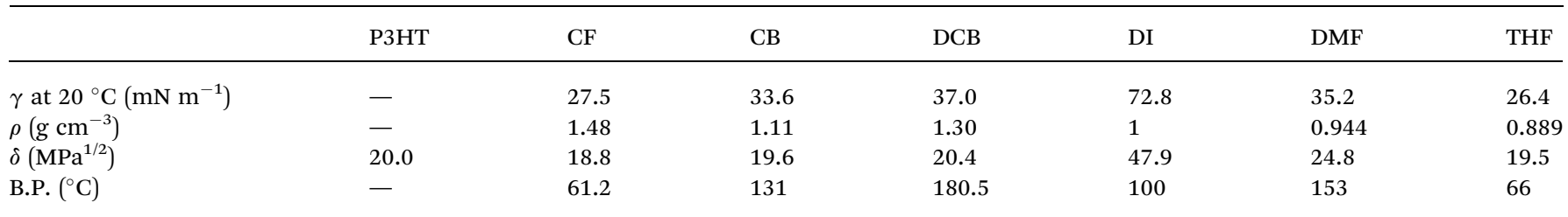

current and field effect mobility as the amount of additive increased. The highest field effect mobility obtained here, 3.91 $\times 10^{-2} \mathrm{~cm}^{2} \mathrm{~V}^{-1} \mathrm{~s}^{-1}$ was obtained from the P3HT device processed using a solvent mixture comprising $10 \mathrm{vol} \% \mathrm{CB}$ in CF (prepared using the mixing method). This value was 380 -fold higher than the value obtained from a device processed from the homogeneous CF solvent, $1.03 \times 10^{-4} \mathrm{~cm}^{2} \mathrm{~V}^{-1} \mathrm{~s}^{-1}$. On the other hand, the devices processed using the DCB solvent additive (prepared using the mixing method) exhibited less enhanced charge transport properties compared to CF:CB; the field effect mobilities were $6.18 \times 10^{-3} \mathrm{~cm}^{2} \mathrm{~V}^{-1} \mathrm{~s}^{-1}$ and $9.50 \times$ $10^{-3} \mathrm{~cm}^{2} \mathrm{~V}^{-1} \mathrm{~s}^{-1}$ for the addition of $5 \mathrm{vol} \%$ and $10 \mathrm{vol} \%$, respectively. The values of field effect mobility, on-off ratio, and $V_{\text {th }}$ are listed for all fabricated devices in Table S1. $\dagger$ The higher on-off ratios and field effect mobility were resulted from improved on-current level. The solvent mixture prepared by the mixing method was more effective at improving the hole field effect mobility than the floating method, which in good agreement with the crystallinity trend confirmed using UV-vis spectroscopy. These field effect mobility values were correlated with the P3HT crystallinities, as confirmed by UV-vis spectroscopy, indicating that a high polymer crystallinity enhanced the charge transport properties of the film.

\subsection{Development of a biphasic system using a solvent additive}

The film formation mechanism from a solvent mixture in a biphasic dip-coating system was explored. The biphasic system prepared using the solvent adding processes, such as the floating method and the mixing method, is illustrated schematically (Fig. 6). The critical solvent mixture factor to consider is the Hansen solubility parameter (HSP), which can be used to predict the compatibility between solvents. Liquids with similar HSPs are expected to be miscible, and polymers will dissolve in solvents with similar HSPs that are not too different from their own. ${ }^{38}$ The HSP is a measure of the solubility and dispersity. It has been widely used to analyze the properties of solvent mixtures and polymer solutions. The HSP difference between a solvent additive and a main solvent is critical to determining how these components mix in the solution state and affect polymer crystallization. ${ }^{39}$ Table 1 lists the differences between the HSP values of the solvents in the biphasic systems: the HSP difference between $\mathrm{CB}$ and $\mathrm{CF}$ was the smallest, $0.8 \mathrm{MPa}^{1 / 2}$, whereas that between CF and DCB was $1.6 \mathrm{MPa}^{1 / 2}$. Thus, the compatibility for intermixing is better for $\mathrm{CB}$ and $\mathrm{CF}$ than for DCB and CF. These calculations provide insight into the biphasic separation behaviors of the solvent mixtures $\mathrm{CF}: \mathrm{CB}$ and CF:DCB.
A system comprising a floating solvent additive, $\mathrm{CB}$, on a main solvent, $\mathrm{CF}$, had a small HSP difference, which enabled the floating solvent additive to gradually intermix into the underlying P3HT solution and induce P3HT crystallization during the dip-coating process. A floating solvent additive DCB displayed a large HSP difference with CF, which inhibited DCB diffusion into the P3HT solution and hampered P3HT crystallization, producing inhomogeneous film formation. Even over longer period of time, we confirm that the floating secondary solvent does not seem to diffuse well to form an intermixed phase gradient with the P3HT-solution/water phase, resulting in a weaker crystallization compared to the mixing method.

A biphasic system prepared through the mixing method, in which a solvent additive $\mathrm{CB}$ was mixed with $\mathrm{CF}$, displayed a small HSP difference between CB and CF, resulting in good intermixing between the solvent additive $\mathrm{CB}$ and the $\mathrm{P} 3 \mathrm{HT}$ molecules due to the high miscibility with CF. Strong P3HT crystallization and homogeneous film formation were induced during the dip-coating process. The mixture of the solvent additive DCB and CF, which had a relatively high HSP difference, induced agglomerated DCB diffusion into the solution state. The forced mixing induced strong P3HT crystallization compared to the floating method, as observed in the UV-vis absorption spectra and device characteristics obtained from the solvent mixture CF:DCB.

\section{Conclusions}

We developed a biphasic dip-coating method with water as a reusable bottom phase solvent for fabricating highly crystalline P3HT thin film transistors. We investigated the effect of the solvent additive on the polymer crystallinity and film formation, which depended strongly on the specific solvent addition method (floating or mixing). The UV-vis absorption properties of the processed films revealed that the solvent additive evaporation rate and the miscibility between the additive and the main solvent affected film formation and polymer crystallization during the dip-coating process. The enhanced polymer crystallinity in the dip-coated films obtained from the solvent mixture was attributed to the extended solvent drying time, which provided sufficient time for the P3HT molecules to selfassemble. These characteristics were consistent with the corresponding device performances. The device processed from a CB:CF solvent mixture exhibited the highest average field effect mobility, $0.0391 \mathrm{~cm}^{2} \mathrm{~V}^{-1} \mathrm{~s}^{-1}$. It should be noted that this method might be used to prepare uniform films over large substrates and to promote long-range ordering of $\mathrm{P} 3 \mathrm{HT}$ crystals without producing environmentally hazardous waste. This 
study provides a promising innovation in the development of low-cost large-area flexible electronics.

\section{Conflicts of interest}

There are no conflicts to declare.

\section{Acknowledgements}

This research was supported by the Incheon National University Research Grant in 2016.

\section{References}

1 S. R. Forrest, Nature, 2004, 428, 911.

2 P. Heremans, A. K. Tripathi, A. de Jamblinne de Meux, E. C. P. Smits, B. Hou, G. Pourtois and G. H. Gelinck, Adv. Mater., 2016, 28, 4266.

3 S. Mandal and Y.-Y. Noh, Semicond. Sci. Technol., 2015, 30, 064003.

4 P. Lin and F. Yan, Adv. Mater., 2012, 24, 34.

5 A. C. Arias, J. D. MacKenzie, I. McCulloch, J. Rivnay and A. Salleo, Chem. Rev., 2010, 110, 3.

6 K. Fukuda and T. Someya, Adv. Mater., 2017, 29, 1602736.

7 C. D. Dimitrakopoulos and P. R. L. Malenfant, Adv. Mater., 2002, 14, 99.

8 S. Riera-Galindo, A. Tamayo and M. Mas-Torrent, ACS Omega, 2018, 3, 2329.

9 D. Khim, H. Han, K.-J. Baeg, J. Kim, S.-W. Kwak, D.-Y. Kim and Y.-Y. Noh, Adv. Mater., 2013, 25, 4201.

10 E. Sowade, E. Ramon, K. Y. Mitra, C. Martínez-Domingo, M. Pedró, J. Pallarès, F. Loffredo, F. Villani, H. L. Gomes, L. Terés and R. R. Baumann, Sci. Rep., 2016, 6, 33490.

11 S. Zhang, L. Ye, H. Zhang and J. Hou, Mater. Today, 2016, 19, 533.

12 A. Facchetti, Mater. Today, 2007, 10, 28.

13 Y. Wang, H. Tatsumi, R. Otsuka, T. Mori and T. Michinobu, J. Mater. Chem. C, 2018, 6, 5865.

14 X. Tang and X. Yan, J. Sol-Gel Sci. Technol., 2017, 81, 378.

15 M. Faustini, G. L. Drisko, C. Boissiere and D. Grosso, Scr. Mater., 2014, 74, 13.

16 K. Wu, H. Li, L. Li, S. Zhang, X. Chen, Z. Xu, X. Zhang, W. Hu, L. Chi, X. Gao and Y. Meng, Langmuir, 2016, 32, 6246.

17 B. Wang, T. Zhu, L. Huang, T. L. D. Tam, Z. Cui, J. Ding and L. Chi, Org. Electron., 2015, 24, 170.

18 L. Li, P. Gao, W. Wang, K. Müllen, H. Fuchs and L. Chi, Angew. Chem., Int. Ed., 2013, 52, 12530.
19 G. Wang, T. Hirasa, D. Moses and A. J. Heeger, Synth. Met., 2004, 146, 127.

20 T. Mori, T. Oyama, H. Komiyama and T. Yasuda, J. Mater. Chem. C, 2017, 5, 5872.

21 M. Li, C. An, W. Pisula and K. Müllen, Small, 2014, 10, 1926.

22 D. R. Ceratti, B. Louis, X. Paquez, M. Faustini and D. Grosso, Adv. Mater., 2015, 27, 4958.

23 Y.-J. Kim, H.-T. Jung, C. W. Ahn and H.-J. Jeon, Adv. Mater. Interfaces, 2017, 4, 1700342.

24 S. Brixi, O. A. Melville, N. T. Boileau and B. H. Lessard, J. Mater. Chem. C, 2018, 6, 11972.

25 C. Reichardt and T. Welton, Solvents and Solvent Effects in Organic Chemistry, 3rd edn, Wiley-VCH Publishers, 2003.

26 P.-G. de Gennes, F. Brochard-Wyart and D. Quere, Capillarity and Wetting Phenomena, Springer, New York, 2004.

27 S.-M. Jin, I. Kim, J. A. Lim, H. Ahn and E. Lee, Adv. Funct. Mater., 2016, 26, 3226.

28 J. Jang, S. Nam, K. Im, J. Hur, S. N. Cha, J. Kim, H. B. Son, H. Suh, M. A. Loth, J. E. Anthony, J.-J. Park, C. E. Park, J. M. Kim and K. Kim, Adv. Funct. Mater., 2012, 22, 1005.

29 J. Y. Na, M. Kim and Y. D. Park, J. Phys. Chem. C, 2017, 121, 13930.

30 J. W. Jeong, G. Jo, S. Choi, Y. A. Kim, H. Yoon, S.-W. Ryu, J. Jung and M. Chang, ACS Appl. Mater. Interfaces, 2018, 10, 18131.

31 P. J. Brown, D. S. Thomas, A. Köhler, J. S. Wilson, J. S. Kim, C. M. Ramsdale, H. Sirringhaus and R. H. Friend, Phys. Rev. B: Condens. Matter Mater. Phys., 2003, 67, 642031.

32 M. Urien, L. Bailly, L. Vignau, E. Cloutet, A. de Cuendias, G. Wantz, H. Cramail, L. Hirsch and J.-P. Parneix, Polym. Int., 2008, 57, 764.

33 M. J. Rosen and J. T. Kunjappu, Surfactants and Interfacial Phenomena, 4th edn, Wiley, 2012.

34 L. J. Richter, D. M. DeLongchamp, F. A. Bokel, S. Engmann, K. W. Chou, A. Amassian, E. Schaible and A. Hexemer, Adv. Energy Mater., 2015, 5, 1400975.

35 C. McDowell, M. Abdelsamie, K. Zhao, D.-M. Smilgies, G. C. Bazan and A. Amassian, Adv. Energy Mater., 2015, 5, 1501121.

36 C. M. Hansen, Hansen Solubility Parameters - A User's Handbook, 2nd edn, CRC Press, Boca Raton, 2007.

37 L. Landau and B. Levich, Acta Physicochim. URSS, 1942, 17, 42.

38 C. M. Hansen and A. L. Smith, Carbon, 2004, 42, 1591.

39 G. W. Kim, Y. J. Jang, M. Kim and Y. D. Park, J. Mater. Chem. $C, 2018,6,8353$. 